\title{
Do we really need sub-micron resolution to analyse single cell molecular features through vibrational spectroscopy? A pilot study using Plasmodium falciparum-infected Human Erythrocytes
}

\section{Agnieszka Banas ( $\nabla$ slsba@nus.edu.sg )}

National University of Singapore https://orcid.org/0000-0001-6268-1081

Krzysztof Banas

National University of Singapore

Trang Thi Thu Chu

National University of Singapore

Renugah Naidu

National University of Singapore

Paul Edward Hutchinson

National University of Singapore

Rupesh Agrawal

National Healthcare Group Eye Institute

Michael Lo

Photothermal Spectroscopy Corp

Mustafa Kansiz

Photothermal Spectroscopy Corp

Rajesh Chandramohanadas

National University of Singapore

Mark Breese

National University of Singapore

Article

Keywords: FTIR microspectroscopy, O-PTIR, AFM-IR, single cell, malaria

Posted Date: March 10th, 2021

DOl: https://doi.org/10.21203/rs.3.rs-294029/v1

License: (c) (i) This work is licensed under a Creative Commons Attribution 4.0 International License.

Read Full License 
Version of Record: A version of this preprint was published at Communications Chemistry on September 9th, 2021. See the published version at https://doi.org/10.1038/s42004-021-00567-2. 


\section{Abstract}

Malaria is one of the major life-threatening diseases to afflict humanity with an estimated 228 million cases worldwide in 2018. There exists no approved Malaria vaccine on the market yet, partly due to the complexity of the parasite life cycle and the vast repertoire of polymorphic proteins they express during different stages of development.

In this work, we have tested two emerging spectroscopic approaches: Optical Photothermal Infrared (OPTIR) spectroscopy and Atomic Force Microscopy combined with infrared spectroscopy (AFM-IR) in contrast to the more traditional Fourier Transform InfraRed (FTIR) microspectroscopy which has emerged recently as a new promising tool to provide label-free analysis of cell and tissue sections. Examples of chemical spatial distributions of selected bands and spectra for Plasmodium falciparum infected RBCs collected with the three modalities are presented and compared together with advantages and limitations of each method. Based on these results, it appears that O-PTIR and AFM-IR techniques can be explored as powerful tools for the analysis of cells and ipso facto, these methods can help in better understanding complex processes occurring within heterogeneous objects such as infected RBC due to their superior spatial resolution in comparison with traditional approaches for infrared spectroscopic characterization.

\section{Introduction}

Malaria is one of the most common and life-threatening diseases that continues to affect mankind. Every year, an estimated 400,000 deaths [1] occur because of this mosquito-borne infectious disease. Since 2000, the rate of new malaria vaccine trials registered at ClinialTrials.gov has remained steady at about 10 trials each year [2]. So far, only one of them, the pre-erythroctic vaccine (PEV) product RTS,S/AS01E has proven its safety and effectiveness in reduction of clinical malaria cases in African children. Up to now, the world is still waiting for optimally developed medications to fight the scourge of malaria. One of the hypotheses claims that malaria vaccine development is slowed down by the complexity of the parasite's life cycle and its vast repertoire of polymorphic proteins.

It seems that early diagnosis is still the key to start immediate treatment and through this to reduce malaria-associated deaths. Currently, methods such as microscopic examination of infected blood [3,4], gene amplification techniques $[5,6]$ or serological detection tests [7] are relied up on clinical diagnosis of malaria. Some of these techniques require sophisticated instruments, expensive reagents or trained personnel, whereas microscopic methods are easy to perform, but are prone to human errors.

Amongst the various species of malaria parasites, Plasmodium falciparum is responsible for most malaria-associated deaths. Sporozoite stage of the parasite enters the human body through the bite of infected mosquito and proliferate asymptomatically into merozoites upon reaching the liver (the preerythrocytic hepatic stage). Later, the merozoites invade the red blood cells (RBC) to announce $\sim 48$ h-long intraerythrocytic developmental cycle (IDC). The very first stage of this reproduction is constrained to the parasitophorous vacuole (PV), a structure allowing a parasite to grow within cell and at the same time 
being protected from the cell defence mechanisms [8]. The young parasites also known as the "ring" stage have low metabolic activity. To facilitate its development, erythrocyte cytoplasm containing haemoglobin is ingested into an acidic digestive vacuole (DV). Hemoglobin is digested in these compartments; this process leaves insoluble reactive products due to the release of heme, which is neutralized by the formation of $\beta$-hematin that biocrystallizes to the chemically inert hemozoin $(\mathrm{Hz})$, also known as the malaria pigment $[9,10]$. DV takes a crucial role in parasite development, it is also a place for amino acid transportation, oxygen radicals detoxification and drug accumulation [11,12]. Along with parasite development, a single large DV is formed at the late ring stage and increases its size in the next trophozoite stage when maximum haemoglobin digestion is observed. In the next IDC stage, schizont, the DV shrinks in size and hemozoin is released into the blood circulation.

Parasites have unique biochemical signatures representing their cellular composition (nucleic acids, lipids, proteins) which can undergo dynamic changes during the IDC. Significant chemical differences between infected and uninfected RBCs suggest that spectroscopic methods could be used to analyze the parasite's developmental process.

Label-free techniques such as Raman imaging microscopy has been tested as a potential method to diagnose plasmodium infections on the basis of strong scattering from the $\mathrm{Hz}$ pigment [13]. However, a rather low signal to noise ratio, high autofluorescence and potential photodamage limits its effectiveness in revealing molecular structures of infected single RBCs Another commonly used method for analysis of plasmodium infected RBCs is FTIR (Fourier Transform InfraRed) microspectroscopy, a method known since the middle of 20th century and broadly applied in biomedical research. FTIR microspectroscopy probes intrinsic molecular vibrational frequencies of bonds between molecules present in a sample. It is worth emphasizing that IR light used for the experiments is too weak to cause any cell damage. Since vibrational frequencies depend on the parameters' characteristic of the molecular structures, they can provide valuable information on cellular biochemical changes through relative quantification of lipids, proteins, carbohydrate etc, as reflected by published literature discussing analysis of cells by means of FTIR spectroscopy [14-16].

However, it is worth emphasizing that there are shortcomings associated with FTIR, which may prevent its use in single cell-based studies. Theoretically, single cells can be analysed either when apertures are used in the IR microscope or when the IR microscope is equipped with a 2D Focal Plane Array (FPA) detector. Aperture-based systems considerably limit the light throughput, and hence significantly degrade spectral signal-to-noise (sensitivity), as the aperture masks throw away most of the incoming infrared light. More advanced FTIR microscopes with FPA detectors remove the need for apertures, with the FPA pixels themselves defining measured area. A FPA detector is composed of many basic detector units recording a full FTIR spectrum within the interval $4000-900 \mathrm{~cm}^{-1}$ from a defined spot (few $\mu \mathrm{m}^{2}$ ) on the sample. However, the amount of light absorbed in the IR region entering the single detector unit is small, leading to noisy spectra. Widely-used FPA detectors composed of 128 horizontal pixels and 128 vertical pixels working with $15 x$ objective (Bruker $0.4 \mathrm{NA}$ ) have an effective pixel size of 2.7 by $2.7 \mu \mathrm{m}^{2}$, however it does not mean that during experiments chemistry from that spot is recorded - it is only a "pixel size." Pixel size 
must not be confused with the spatial resolution, as it is one of the most critical measurements parameters in FTIR microspectroscopy [17]. The spatial resolution is restricted by the so-called diffraction limit and can be calculated using Rayleigh criterion, which depends on the wavelength. For $15 x$ objective mentioned earlier, simple calculations show that for selected wavenumbers much larger values than 2.7 $\mu \mathrm{m}$ have been obtained: $15 \mu \mathrm{m}$ (at $1000 \mathrm{~cm}^{-1}$ ), $9.2 \mu \mathrm{m}\left(\right.$ at $1650 \mathrm{~cm}^{-1}$ ), and $5.4 \mu \mathrm{m}$ (at $2800 \mathrm{~cm}^{-1}$ ). It means that the response from the system comes from an area much bigger than the pixel size, leading to spatial averaging of biochemical heterogeneities across areas of up to $\sim 15 \mu \mathrm{m}$ within the single cell, thus severely limiting intra-cellular imaging.

With a commercial malaria vaccine remaining elusive, there is still a need for understanding the interactions of the plasmodium parasites with its host cells during the complex multistage life cycle. As mentioned earlier, DV takes a crucial role in parasite development. Studying the changes the cell undergoes can also have the potential to identify key biological and physiological processes that could lead to new and improved antimalarial treatments. If vibrational spectroscopy is being considered as a potential method of examining infected RBC, according to the authors of this paper, it is necessary to remember that detailed analysis of parasite-host interactions should be performed at the microscale or even the nanoscale. Multiple small DVs which are characteristic of the early and mid-stage rings have a diameter of $150-300 \mathrm{~nm}$ [18], at the trophozoite stage, when haemoglobin degradation is noticeable, DV has a diameter of up to $2.2 \mu \mathrm{m}$, and later in schizogony stage it decreases in size to 0.8-1.2 $\mu \mathrm{m}[19,20]$.

The last decade has seen impressive progress in the development of new technique based on the interpretation of vibrational frequencies of bonds present in molecules. One of them is AFM-IR (Atomic Force Microscopy - Infrared) spectroscopy. This technique combines the power of infrared absorption spectroscopy for non-destructive, label-free chemical identification with the nanoscale resolution obtained by atomic force microscopy and can be successfully applied to study the composition of cells at the nanoscale [21, 22]. According to the authors' knowledge, AFM-IR has been already used to analyse malaria infected RBCs [23]. Another novel and highly versatile technique, Optical Photothermal Infrared (O-PTIR) spectroscopy, has captured recent attentions for its high versatility and offers submicron resolution for IR imaging and spectroscopy [24]. This method is also label-free, requires no special sample preparation steps, operates in an easy-to-use far-field mode (non-contact) and offers submicron resolution for IR imaging and spectroscopy [24].

In this paper, we will determine the relevancy of sub-micron resolution towards the analysis of Plasmodium falciparum-infected human erythrocytes by means of vibrational spectroscopy. Comparison of two evolving techniques (O-PTIR and AFM-IR) against the more traditional FTIR microspectroscopy based on results obtained for the same infected RBC will be presented along with the advantages and limitations of each method. Infected RBC (iRBC) was selected as a subject of our analysis due to its heterogeneity related to the parasite invasion. Our work is mainly focused on illustrating all the benefits (if any) of analysing iRBC content with sub-micron resolution. Herein, we deliberately refrain from any biochemical interpretation of the obtained results. As we are presenting comparative data from the exact 
same iRBC in the paper using three techniques, however we have measured/compared about 20 cells and conclusions presented in the paper are a cumulative sum of our consistent observations.

To our knowledge, this is the first comprehensive investigation aimed to classify, compare and identify the best method, which could be used for the spectroscopic characterisation of single cells in mid-IR region in follow-up studies.

\section{Materials And Methods}

All spectroscopic/imaging experiments were performed at the branch of the upgraded ISMI beamline (at the Singapore Synchrotron Light Source) by means of:

1. Hyperion 3000 (IR microscope) equipped with FPA detector (128 pixels by 128 pixels) attached to Vertex 80v (both Bruker) spectrometer - for FTIR microspectroscopy,

2. mIRage microscope produced by the Photothermal Spectroscopy Corp (PSC) - for the O-PTIR submicron IR characterization,

3. nanolR3 system produced by Bruker Nano - for nanoscale AFM-IR absorption spectroscopy.

\section{Preparation of plasmodium-infected blood samples}

3D7 strain of $P$. falciparum parasites grown in human blood (purchased from Interstate Blood Bank, USA) were used in all experiments, in agreement with approved protocols of Singapore University of Technology \& Design. Synchrony of the cultures was achieved by frequent selection of ring-stage infections using sorbitol treatment. Trophozoite stage parasites ( 30 hours post-invasion) were used in all experiments reported in this work.

Infected RBCs (around 5\% parasitemia) were washed once in 1X PBS followed by fixation in 2\% PFA for $15 \mathrm{~min}$. Fixed cells were smeared on $\mathrm{CaF}_{2}$ windows (Crystran, UK) and air-dried for further use. Samples were first examined under 4x, 10x and 40x objective lens (Leica DM750) and microscopic images were captured by Leica ICC50W digital camera. Since the smears were not stained, the infected cells were recognized based on the typical black hemozoin dots of trophozoite stage parasites. Infected cells were selected and labelled from high contrast 40x microscopic images and the corresponding 10x and $4 x$ images. Further scans by FTIR, O-PTIR or AFM-IR were focused on these selected area and cells.

\section{Details of the experiments FTIR microspectroscopy}

Analysis of plasmodium-infected RBCs were performed by means of FTIR microspectroscopy in transmission mode using Bruker Hyperion 3000 IR microscope with a FPA detector attached to the Vertex

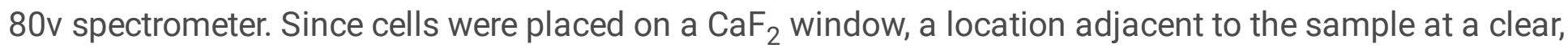
clean spot on that window was used for background signal collection. 
An IR objective with $15 \times$ magnification was used that provides a pixel size equal to $2.7 \mu \mathrm{m}^{2}$ (with 128 by 128 pixels in each direction of the FPA detector). The number of co-added scans were tested in order to ensure sufficient signal-to-noise ratio. 1024 scans provided a balance between good quality spectra within a reasonable amount of time (32 mins).

A spectral range of 3845 to $900 \mathrm{~cm}^{-1}$ was set with a spectral resolution of $8 \mathrm{~cm}^{-1}$. Zero filling factor was set to 2 and Blackman-Harris 3-Term apodization function with phase resolution 32 and power phase correction mode was selected for converting measured interferograms to final spectra.

From a single experiment, 16,384 spectra were collected from an area approximately equivalent to $345 \mathrm{x}$ $345 \mu \mathrm{m}^{2}$.

\section{Sub-micron IR characterization (O-PTIR)}

O-PTIR sub-micron IR characterization was carried out using a state-of-the-art mIRage microscope (PSC, USA). As a source of IR radiation, a tuneable pulsed mid-IR Quantum Cascade Laser (QCL) was used during experiments operating with $300 \mathrm{~ns}$ pulses at a repetition rate of up to $100 \mathrm{kHz}$. The selected area within the sample was illuminated, which caused small photothermal perturbations in the form of rapid conversion of photon energy into heat, lasting for a few picoseconds. This phenomenon was probed by a green $(532 \mathrm{~nm})$ laser, position of which was co-located with the IR light. Here, the visible probe beam would detect the localised reflectivity changes on the sample surface, hence 0-PTIR's sensitivity was applicable only to the materials' IR absorptivity at a given wavenumber. The laser power levels of the IR and probe were each set to approximately $1-5 \mathrm{~mW}$ per spectrum. O-PTIR measurements were done in a non-contact reflection mode while providing FTIR transmission-like infrared spectra that were artefactfree (without Mie scattering effects). Available at the ISMI beamline, QCL comprised of 4 chips and enabled spectra collection within the mid-IR fingerprint region of $1800-800 \mathrm{~cm}^{-1}$. The number of coadded scans per spectrum was 20 to maintain good signal to noise ratio. The effective spectral resolution was approximately $6 \mathrm{~cm}^{-1}$.

The spatial resolution in the 0-PTIR method is dependent on the spot size of the short wavelength green laser. During the experiments, its size was maintained below $1 \mu \mathrm{m}$ to deliver submicron spatial resolution.

As a first step in preparation of the system for experiments, the background signal was recorded to take into account the influence of the IR laser power distribution on final spectrum.

Before all experiments, the system was wavenumber calibrated using a test sample provided by the manufacturer (cross-sections of PMMA and PS beads embedded in epoxy resin).

Prior to sample analysis, microscope photographic documentation of cells was performed with different objectives: low magnification visible (refractive objective) 10x, 0.3NA (working distance of about $15 \mathrm{~mm}$ ) and high magnification (reflective Cassegrain objective) 40x, 0.78NA (working distance of about $8 \mathrm{~mm}$ ). 
The latter was also used for data (spectra and single wavenumber images) collection, which were saved and processed in PTIRStudio 4.0 software supplied with the instrument.

Samples to be analysed were placed on the automated scanning stage in the sample chamber; the entire instrument was purged with dry nitrogen during all experiments in order to eliminate water vapour absorption.

During experiments apart from single spectra taken from selected areas within single cells, single wavenumber images were also collected.

\section{Nanoscale IR Characterisation (AFM-IR experiments)}

$\mathrm{CaF}_{2}$ window with RBCs fixed on it, has been mounted on a steel holder and analysed within the $1800-800 \mathrm{~cm}^{-1}$ spectral range with a resolution of about $6 \mathrm{~cm}^{-1}$ (spectral point density equal to $2 \mathrm{~cm}^{-1}$ ) by means of the nanoIR3 instrument (Bruker Nano, USA); Analysis Studio 3.16 provided with the instrument was used to collect all experimental data. The same QCL laser (described earlier) was used as the source of IR. The samples were scanned in contact mode at a scan rate of $0.02 \mathrm{~Hz}$ using a goldcoated AFM tip with an apex of nominal curvature radius (R) of sub-30 nm (Anasys Instruments, USA). The cantilever had a nominal spring constant of $0.07-0.4 \mathrm{~N} / \mathrm{m}$ and a resonance frequency of $13 \pm 4 \mathrm{kHz}$ while in a "free-state." Rapid laser pulses (QCL) (a few nanoseconds duration, with $100 \mathrm{kHz}$ repetition rate) tuned to a corresponding absorption bands occurring in the samples, caused rapid thermal expansions thereby resulting in an oscillation of the AFM tip placed in contact with the material. The obtained spectrum was the amplitude of the cantilever oscillation, which is proportional to the absorption coefficient of the sample at different wavenumber values after normalizing against the background.

Prior to experiments, nanolR3 system has been calibrated using a test sample (the same as for O-PTIR technique) provided by the manufacturer. Background has been collected to compensate the input from the atmospheric gases and to take into account the power distribution of the IR laser delivered on the sample.

For collecting data, the second harmonics of the cantilever oscillation mode was chosen for optimizing the cantilever ringdown signal centred at its frequency of $\sim 180 \mathrm{kHz}$ with a frequency window of $50 \mathrm{kHz}$. For each spectrum 16 scans were co-averaged. For chemical imaging, the scanning was performed with 400 points in the $x$ and 100 points in the $y$ direction co-averaged over 128 ringdowns. It is worth noting that with all IR absorption images (for selected wavenumbers), AFM images (height and deflection signals) were simultaneously recorded for the same regions of interest in order to gain information regarding the topography of the sample.

As described earlier, these methods gave us the unique opportunity to analyse exactly the same sample as all these methods can be treated as non-destructive; after every experiment the specimen was left in pristine condition. 
Table 1 presents the summary of experiments carried out on the same iRBC by means of three modalities discussed earlier.

Table 1 Summary of the experiments performed on iRBC sample

\begin{tabular}{|c|c|c|c|}
\hline $\begin{array}{l}\text { Method } \\
\text { Spectral } \\
\text { range } \\
\text { Spatial } \\
\text { resolution }\end{array}$ & $\begin{array}{l}\text { FTIR microspectroscopy } \\
\text { (Hyperion } 3000 \text { ) } \\
3845 \text { to } 900 \mathrm{~cm}^{-1} \\
\text { IR Wavelength dependent, can } \\
\text { be calculated using Rayleigh } \\
\text { criterion; } 15 \mu \mathrm{m} \text { (at } 1000 \mathrm{~cm}^{-1} \text { ), } \\
9.2 \mu \mathrm{m} \text { (at } 1650 \mathrm{~cm}^{-1} \text { ), and } 5.4 \\
\mu \mathrm{m} \text { (at } 2800 \mathrm{~cm}^{-1} \text { ) }\end{array}$ & $\begin{array}{l}\text { O-PTIR } \\
\text { (mIRage) } \\
1800-800 \mathrm{~cm}^{-1} \\
<1 \mu \mathrm{m} \text { resolution }\end{array}$ & $\begin{array}{l}\text { AFM - IR } \\
\text { (nanoIR3) } \\
1800-800 \mathrm{~cm}^{-1} \\
\sim 40 \mathrm{~nm} \text { resolution }\end{array}$ \\
\hline $\begin{array}{l}\text { Single } \\
\text { spectrum }\end{array}$ & $\begin{array}{l}128 \times 128=16384 \text { spectra } \\
\text { collected in each experiment } \\
\text { (FPA detector) }\end{array}$ & $\begin{array}{l}\text { Collected from } \\
\text { selected spots }\end{array}$ & $\begin{array}{l}\text { Collected from } \\
\text { selected spots }\end{array}$ \\
\hline $\begin{array}{l}\text { IR absorption } \\
\text { images = } \\
\text { Distribution } \\
\text { of certain } \\
\text { wavenumbers }\end{array}$ & $\begin{array}{l}\text { Done as a post-processing } \\
\text { (integrals calculation) of } \\
\text { collected array of the spectra }\end{array}$ & $\begin{array}{l}\text { Collected for } \\
\text { selected } \\
\text { wavenumbers; one } \\
\text { image = chemical } \\
\text { distribution for one } \\
\text { wavenumber }\end{array}$ & $\begin{array}{l}\text { Collected for } \\
\text { selected } \\
\text { wavenumbers; one } \\
\text { image = chemical } \\
\text { distribution for one } \\
\text { wavenumber }\end{array}$ \\
\hline $\begin{array}{l}\text { Topography } \\
\text { information }\end{array}$ & Not available & $\begin{array}{l}\text { Collected in the form } \\
\text { of mIRage laser } \\
\text { reflectivity image } \\
\text { (the relative } \\
\text { reflectivity of the } \\
\text { green laser) }\end{array}$ & $\begin{array}{l}\text { Collected in form of } \\
\text { AFM images (height } \\
\text { and deflection } \\
\text { channels) }\end{array}$ \\
\hline
\end{tabular}

\section{Data Availability}

The datasets generated during and/or analysed during the current study are available from the corresponding author on reasonable request.

\section{Results}

All methods discussed in this paper probe intrinsic molecular vibrational frequencies of bonds between molecules present in a sample; these absorption bands are displayed as a function of wavenumber [ $\mathrm{cm}^{-}$ $\left.{ }^{1}\right]$. 
A typical (vibrational) spectrum of a biological sample shows several absorbance bands associated with the main macromolecules found in most biological materials, namely amide bands from proteins, membrane lipids $\left(\mathrm{CH}_{\mathrm{x}}\right.$ stretching and deformation), nucleic acids (phosphate stretches), carbohydrates (CO stretching) and phospholipids (Table 2).

Tab. 2 Most common IR bands found in the FTIR spectra of RBCs and P. falciparuminfected RBCs [25-27]

\begin{tabular}{|l|l|l|}
\hline Band & Assignment & Major contributions \\
\hline 2953 & $\nu_{\text {as }} \mathrm{CH}_{3}$ & Lipids, Proteins \\
\hline 2920 & $\nu_{\mathrm{as}} \mathrm{CH}_{2}$ & Lipids \\
\hline 2864 & $\nu_{\text {sym }} \mathrm{CH}_{3}$ & Lipids, Proteins \\
\hline 2852 & $\nu_{\text {sym }} \mathrm{CH}_{2}$ & Lipids \\
\hline 1742 & $\nu \mathrm{C}=\mathrm{O}$ ester carbonyl & Lipids \\
\hline $1704-1710$ & $\nu \mathrm{C}=\mathrm{O}$ & Hz (hemozoin) \\
\hline $1660-1640$ & Amide I & Proteins \\
\hline $1545-1525$ & Amide II & Proteins \\
\hline 1452 & & Lipids \\
\hline 1391 & $\begin{array}{l}\nu \mathrm{COO}_{2} \text { of fatty acids and amino acid } \\
\text { side chains }\end{array}$ & $\begin{array}{l}\text { Lipids/ } \\
\text { Proteins }\end{array}$ \\
\hline $1310-1250$ & amide III & Proteins \\
\hline 1244 & $\nu_{\text {as }} \mathrm{PO}_{2}$ & RNA \\
\hline $1207-1220$ & $\nu \mathrm{C}-\mathrm{O}$ & Hz (hemozoin) \\
\hline $960-1200$ & $\nu \mathrm{C}-\mathrm{O}, \nu \mathrm{C}-\mathrm{C}, \nu \mathrm{P}-\mathrm{O}$ & DNA, saccharides, lipids \\
\hline
\end{tabular}


The biochemical composition (and therefore molecular signatures that are extracted by spectroscopic techniques) of $P$. falciparum-infected RBCs (iRBC) are bound to be different as a result of parasite development (for example, synthesis of Plasmodium specific proteins, haemoglobin degradation, peptide and/or amino acid transportation, heme biocrystalization, etc [12]) and/or arising from metabolism (such as iron deficiency, inflammation [28]). Hence, some differences within collected spectra for iRBC in comparison to the spectra collected for healthy cells should be expected. These differences can be manifested in the shape changes such as occurrence of shoulder features in the existing bands and positional shifts of the main absorbance bands in the collected spectra from much localized areas.

In our analysis, infected cells appeared as heterogenous objects making it important to scan multiple areas within each single cell, to check if the existence of parasite "is felt" over the whole cell to the same extent, before any conclusion is drawn.

In order to comparatively present the advantages and limitations of the methods adopted in this work, results from experiments performed for the same infected cell were considered.

First, it is necessary to stress that parasite-infected cells are in the minority (our experiment used samples containing $\sim 5 \%$ infected cells) as can be seen in the representative image (Fig. 1), taken by Leica Optical Microscope with 40x magnification. The diameter of the cells ranges between 8 to $10 \mu \mathrm{m}$.

As mentioned earlier, FTIR microspectroscopy was carried out using FPA detector (128 pixel by 128 pixel); a single pixel element defines a spot size of $2.7 \mu \mathrm{m}^{2}$, though the actual achievable spatial resolution is limited by the wavelength dependent Rayleigh Criterion, which with the microscope configuration used in this study and for the main lipids peaks is about $\sim 5$ microns and up to $\sim 15$ microns of spatial resolution for the saccharide bands at around $1000 \mathrm{~cm}^{-1}$. A full field of view $\left(345 \times 345 \mu \mathrm{m}^{2}\right)$ is analysed in one measurement, as this is the dimension of the full field of view with $15 x$ magnification objective attached to the microscope, it cannot be changed. Fig. 2a depicts the screenshot from the OPUS software (provided with the Bruker instrument); as can be seen, the area selected for experiment (red square) contains not only RBCs, but also empty spaces. Essentially, the data cube of 16384 spectra includes both the biochemistry of the cell and the substrate. A potentially more efficient and higher spatial resolution method to analyse samples that are quite spatially sparsely distributed, might be more "single point" techniques such as O-PTIR and AFM-IR spectroscopies, where analyses can be performed on single cells, deliberately chosen for analysis. Fig. 2c presents an optical image of the sample with the cell of interest in the centre taken with 0-PTIR under high magnification 40x. Finding the cell of interest was a simple and direct procedure for that method. For AFM-IR a cantilever is used to perform analysis and measurements can be done on single cell, but localizing it is not straightforward as the instrument field of view is more limited. Even if the characteristic region of the sample is found (as it is shown in Fig. 2e) it is challenging to ensure that the cell of interest would be analysed as it is hidden under the cantilever shadow. Therefore, generating an initial AFM image of the sampling area is a pre-requisite for capturing and confirming the location of the selected cell (Fig. 2f). 
In order to select the FTIR spectra collected for chosen iRBC, digital zooming is needed. It enables display of the position of single detectors of the FPA, as can be seen in Fig $2 \mathrm{~b}$. The cell of interest is covered by approximately 9 elements of the FPA, some of them extending beyond the cell area into the substrate. As stated in the introduction, O-PTIR and AFM-IR spectroscopies enable analysis of area much smaller than $2.7 \mu \mathrm{m}^{2}$.

As a direct result of the sub-diffraction limited infrared techniques of O-PTIR and AFM-IR, spatially resolved analysis reveals localised chemistry from the same infected cells in far greater detail than the nine (9) FPA-FTIR pixels could. Instead of one FTIR spectrum recorded from the spot of up to 15 microns, around several hundred spectra can be obtained by means of O-PTIR spectroscopy and thousands of spectra for AFM-IR.

It can be argued whether such detailed analysis is needed; the answer depends on the scale of biochemical spatial heterogeneity of the analyzed sample. Fig. 3 depicts the comparison of spectra collected during experiments by means of FTIR microspectroscopy, O-PTIR and AFM-IR.

As previously discussed, the process of data collection varies significantly among these methods, however their resultant spectra can be used to gain information about biochemical constituents of the cells.

FTIR microspectroscopy is represented by nine spectra, which completely span the area of the whole iRBC; while for O-PTIR and AFM-IR six spectra have been shown, they were taken from the points marked in Fig. $2 \mathrm{c}$ and $2 \mathrm{f}$. The points for analysis were randomly selected, the distance between two points lies in the range of $400-900 \mathrm{~nm}$. O-PTIR spectra were collected from areas of $500 \mathrm{~nm}^{2}$, whereas AFM-IR spectra were collected from even smaller areas approximately $40 \mathrm{~nm}^{2}$.

Looking at the spectra collected for iRBC (Fig. 3), it can be seen that the most diverse are spectra obtained by means of either O-PTIR or AFM- IR spectroscopies. Band intensities, their ratios and even shapes and shifts in the positions are clearly visible and highly connected to local biochemical profile.

A noteworthy observation is that all nine FTIR spectra are consistent, with positions and ratios for amide I and II bands are almost the same. This finding is expected as in our work, a FPA detector (128 by 128) and $15 x$ objective were used, so the spatial resolution of that system ranges from 9 to $15 \mu \mathrm{m}$ across the fingerprint region. Pretty much all the spectra from the cell should look similar from averaging a large sampling volume within the cell. Therefore, in this case, the lack of obvious differences indicates that any unique information about biochemical characteristics are attenuated by averaging with neighbouring matters.

To better illustrate the differences, if any, among the collected spectra, hierarchical cluster analysis (HCA) has been performed. Prior to HCA, all spectra were normalized (min-max normalization), otherwise it would be difficult or even impossible to calculate the differences between each pair of the spectra. 
As can be seen, the dendrogram in Fig. 4 clearly shows a hierarchical relationship between the spectra that were chosen for comparison. Spectra were allocated by the algorithm into three groups according to the type of experiments they were part of. FTIR spectra are linked together at very low height that implicates high correlation (or similarity)-For O-PTIR and AFM-IR spectra prominent differences are noticed as representative spectra are joined together further apart in comparison to FTIR spectra.

In vibrational spectroscopic measurements, spectra are the most basic yet most informative component for studying biochemical changes in samples. Apart from spectral information, many scientists rely also on imaging. Spectroscopic imaging does not require usage of additional dyes; different chemical components are visualized based on inherent molecular vibrations arising from them.

In case of FTIR microspectroscopy, a single experiment using a FPA detector can generate a hyperspectral object with 16,384 (array of 128 by 128 ) single spectra. Each spectrum contains information about all absorbance bands present in the sample. By calculating the area under selected bands (for example corresponding to proteins Amide I) image of the particular infrared absorption in space is created. This methodology is quite powerful, taking into account that one experiment can generate a complete range of spectra, which can be later used to obtain spectroscopic images for any selected wavenumber (simply by performing mathematical calculations). Indeed, the speed of measurements could be regarded as the significant asset if only spatial resolution was not so important.

In the case of O-PTIR and AFM-IR spectroscopies, imaging is slower comparing to FTIR microspectroscopy. Due to characteristics of the IR source (QCL), single wavenumber images (also known as discrete frequency images) are captured in the form of 2D area; their size can be set by the analyst, which is very convenient as only the area of the interest is analyzed. Every single point of the image has a unique value corresponding to O-PTIR amplitude or tip oscillation amplitude (in the case of AFM-IR), proportional to the absorbance for the particular wavenumber and the quantity of the material at this spot. In order to obtain a chemical image for another wavenumber, QCL must be tuned to the new wavenumber and the whole procedure must be repeated. Software provided by the manufactures allows the analyst to collect automatically different images at different wavenumbers as a sequence.

Fig. 5 depicts the distributions of Amide l, obtained during analysis of the same cell by means of FTIR microspectroscopy (a), O-PTIR (b) and AFM-IR (c). While we are aware that such a comparison is approximated and simplistic - in the case of FTIR microspectroscopy area under bands of Amide I is presented whereas for O-PTIR and AFM-IR discrete frequency images for $1660 \mathrm{~cm}^{-1}$ are taken into account, this representation is meaningful for the purpose of our work.

FTIR microspectroscopy provides an excellent way to visualize the spatial distribution of cellular constituents (lipids, phospholipids, DNA, etc) $[15,16,23]$. However, looking at the distribution of Amide I from the FTIR measurement presented in Fig. 5 a, it seems that the image is just blurred. Even if the area around the cell of interest can be digitally zoomed in, (Fig. 5 b) it will not help to better visualize the Amide I distribution as it is limited by diffraction limited spatial resolution of the method. The wavelength 
dependence on spatial resolution can provide some improvement in distribution quality, but only for higher wavenumber values $\left(3000-2800 \mathrm{~cm}^{-1}\right)$.

In the case of O-PTIR analysis, the significantly enhanced spatial resolution manifests its presence in an image showing a heterogeneous single cell structure (Fig. 5c). More details are visualized even better in image obtained through AFM-IR analysis (Fig. 5d), owing to the nanoscale resolution of the method. It is worth noting that the protein distribution looks quite different between images obtained by means of 0 PTIR and AFM-IR. This fact can be explained not only by differences in spatial resolution, but also by the way of performing experiments: AFM-IR is more surface specific method, whereas O-PTIR gets signals also from deeper layers of the cell.

Of course, the interpretation of single frequency images can be complicated due to the contribution of thickness variations over the red blood cell at various sampling locations. To see the true molecular contrast, usually the ratio of single frequency images is calculated to normalise for sample thickness. For this article, we focus on comparing the outputs from these three analytical methods. Presenting outcomes of further analysis (spectral differences discussion and their interpretation as well as band ratios calculation) exceeds the scope of this contribution and would be published separately.

\section{Conclusions/summary}

In this paper, we compared three methods: FTIR microspectroscopy, O-PTIR, and AFM-IR spectroscopy that can be used for single cell analysis - in our case- Plasmodium falciparum-infected human RBCs. Potential advantages and limitations of the mentioned methods prompted us to carry out these experiments and to compare between them. Furthermore, if all three discussed methods can deliver similar molecular information, our aim is to prioritize the most economical technique in terms of cost of consumables, manpower and ease of operations and data processing.

As was shown in Figs. 3 and 4 spectra collected by means of FTIR microspectroscopy cannot reveal any meaningful differences at the scale of an infected RBC's size. Therefore, if there is truly no differences, a similar pattern of IR spectra should be seen in results obtained through O-PTIR and AFM-IR spectroscopy. However, various intensities for absorption bands and their shifts in position observed in O-PTIR and AFM-IR spectra can manifest different local biochemistries related to the presence of DVs within the analyzed iRBC. This finding suggests that submicron resolution is indeed required if local biochemical variability within single cell must be checked.

All techniques used in this work, i.e., FTIR microspectroscopy, O-PTIR and AFM-IR spectroscopy, can provide quantitative distribution of bio-molecules such as DNA, carbohydrates, proteins, lipids and in the case of Plasmodium falciparum, hemozoin ( $\mathrm{Hz})$. However, there are essential factors (summarized in Table 3) aim to determine the method's suitability for the analysis of the biochemical landscape of single cells. 
Table 3

Advantages and disadvantages of methods discussed in this manuscript.

FTIR microspectroscopy

Advantages
Thousands of FTIR spectra are collected in the timescale of minutes (16,384 spectra; $8 \mathrm{~cm}^{-1}$ resolution, 1024 scans were collected in 28 minutes)
Disadvantages - Presence of artefacts like Mie scattering in collected spectra

- Diffraction-limited spatial resolution not suitable to analyse objects smaller than 5-15 $\mu \mathrm{m}$; especially for single cell analysis

- Extremely large volumes of data generated

and associated with chemical images (in the order of gigabytes of data per hour)

cryogenic cooling (liquid nitrogen) of the detectors; must be done by trained personnel
O-PTIR spectroscopy

- Sub-micron resolution is suitable for analysis different area within single cell

- 1 spectrum (20 scans) with effective spectral resolution of 6 $\mathrm{cm}^{-1}$ was collected within 3 minutes

- Chemical visualization can be performed with submicron resolution; tiny structures within the sample can be easily presented

- Collected spectra are free from spectral artefacts (e.g. Mie Scattering)

- Depending on the size and resolution of collected images; usually few minutes are needed

- Setting the proper power levels of IR and green (probe) light is needed prior to experiments

- Chemical visualization is done for one wavenumber at a time during one experiment; the process could be automated
AFM-IR

- Nanometer resolution enables for very precise analysis of heterogenous small object such as infected cell

- 1 spectrum (20 scans) with effective spectral resolution of $6 \mathrm{~cm}^{-1}$ is collected within 3 minutes

- Chemical visualization can be performed with nanometer resolution; miniscule structures within the sample can be easily presented
- Time-consuming adjustments and optimization of the system

- Chemical visualization is done for one wavenumber

at a time

- Depending on the size only one cell can be analysed in reasonable time; usually tens of minutes are needed

- As it is a contact technique-special attention must be paid to avoid sample damage 
Based on the presented results, we conclude that the O-PTIR and AFM-IR spectroscopies are preferred choices for analyzing complex samples/cells, such as plasmodium-infected blood cells, offering chemical information pertinent to a heterogeneous environment within single cell.

The sub-micron and nano-resolution offered by these techniques make them ideal modalities for better understanding malaria pathophysiology, where sample availability is limited as it is in the case of $P$. vivax infections. These techniques open new avenues for non-invasive monitoring of biochemical processes occurring within single cells.

\section{Declarations}

\section{Author Contributions}

AB: Contributed to study design, performed FTIR experiments, analysed data and wrote the manuscript; KB: Contributed to study design, performed FTIR experiments, analysed data and wrote the manuscript; TTTC: Contributed to study design, performed plasmodium experiments, contributed to data analysis and assisted with manuscript preparation; RN: Performed plasmodium experiments, contributed to data analysis and assisted with manuscript preparation; PEH: contributed to study design and provided reagents and analysis tools; RA: Contributed to study design and provided reagents and analysis tools; ML: Assisted with data analysis and verification; MK: Assisted with data analysis and verification; RC: Designed the study, Funding and resource acquisition, verified the results, coordinated project, reviewed and edited the manuscript; MB: Funding and resource acquisition, verified the results, reviewed and edited the manuscript.

\section{Competing Interests}

Authors declare no competing financial interests.

\section{References}

1. WHO World Malaria Report 2019, https://www.who.int

2. Duffy, P. E.; Patrick Gorres, J. Malaria Vaccines since 2000: Progress, Priorities Products. npj Vaccines 2020, 5 (1), 1-9. https://doi.org/10.1038/s41541-020-0196-3.

3. Kolaczinski, J.; Mohammed, N.; Ali, I.; Ali, M.; Khan, N.; Ezard, N.; Rowland, M. Comparison of the OptiMAL ${ }^{\circledR}$ Rapid Antigen Test with Field Microscopy for the Detection of Plasmodium Vivax and P. Falciparum: Considerations for the Application of the Rapid Test in Afghanistan. Ann. Trop. Med. Parasitol. 2004, 98 (1), 15-20. https://doi.org/10.1179/000349804225003127.

4. Swarnkar, T.; Pattanaik, P. A. Vision-Based Malaria Parasite Image Analysis: A Systematic Review. Int. J. Bioinform. Res. Appl. 2019, 15 (1), 1. https://doi.org/10.1504/ijbra.2019.10019387.

5. Richard, F. A.; Sehgal, R. N. M.; Jones, H. I.; Smith, T. B. A Comparative Analysis of PCR-Based Detection Methods for Avian Malaria. Parasitol. 2002, 88 (4), 819-822. 
https://doi.org/10.1645/0022-3395(2002)088[0819:ACAOPB]2.0.C0;2.

6. Grabias, B.; Essuman, E.; Quakyi, I. A.; Kumar, S. Sensitive Real-Time PCR Detection of Plasmodium Falciparum Parasites in Whole Blood by Erythrocyte Membrane Protein 1 Gene Amplification. J. 2019, 18 (1), 1-9. https://doi.org/10.1186/s12936-019-2743-9.

7. Rodrigues, M. H. C.; Cunha, M. G.; Machado, R. L. D.; Ferreira, O. C.; Rodrigues, M. M.; Soares, I. S. Serological Detection of Plasmodium Vivax Malaria Using Recombinant Proteins Corresponding to the 19-KDa C-Terminal Region of the Merozoite Surface Protein-1. J. 2003, 2, 1-7. https://doi.org/10.1186/1475-2875-2-1.

8. Wallqvist, A.; Fang, X.; Tewari, S. G.; Ye, P.; Reifman, J. Metabolic Host Responses to Malarial Infection during the Intraerythrocytic Developmental Cycle. BMC Syst. Biol. 2016, 10 (1), 1-18. https://doi.org/10.1186/s12918-016-0291-2.

9. Pagola, S.; Stephens, P. W.; Bohle, D. S.; Kosar, A. D.; Madsen, S. K. The Structure of Malaria Pigment B-Haematin. Nature 2000, 404 (6775), 307-310. https://doi.org/10.1038/35005132.

10. Dilanian, R. A.; Streltsov, V.; Coughlan, H. D.; Quiney, H. M.; Martin, A. V.; Klonis, N.; Dogovski, C.; Boutet, S.; Messerschmidt, M.; Williams, G. J.; Williams, S.; Phillips, N. W.; Nugent, K. A.; Tilley, L.; Abbey, B. Nanocrystallography Measurements of Early Stage Synthetic Malaria Pigment. Appl. Crystallogr. 2017, 50 (5), 1533-1540. https://doi.org/10.1107/S1600576717012663.

11. Olliaro, P. L.; Goldberg, D. E. The Plasmodium Digestive Vacuole: Metabolic Headquarters and Choice Drug Target. Today 1995, 11 (8), 294-297. https://doi.org/10.1016/0169-4758(95)80042-5.

12. Wunderlich, J.; Rohrbach, P.; Dalton, J. P. The Malaria Digestive Vacuole. Biosci. - Sch. 2012, 4 S(4), 1424-1448. https://doi.org/10.2741/s344.

13. Wood, B. R.; Hermelink, A.; Lasch, P.; Bambery, K. R.; Webster, G. T.; Khiavi, M. A.; Cooke, B. M.; Deed, S.; Naumann, D.; McNaughton, D. Resonance Raman Microscopy in Combination with Partial DarkField Microscopy Lights up a New Path in Malaria Diagnostics. Analyst 2009, 134 (6), 1119-1125. https://doi.org/10.1039/b822603b.

14. Moore, D. S.; Jepsen, P. U.; Volka, K. Principles of Vibrational Spectroscopic Methods and Their Application to Bioanalysis. Spectrosc. Second. Enlarg. Ed. 2014, 3-4, 1037-1078. https://doi.org/10.1002/9783527654703.ch27.

15. Perez-Guaita, D.; Andrew, D.; Heraud, P.; Beeson, J.; Anderson, D.; Richards, J.; Wood, B. R. High Resolution FTIR Imaging Provides Automated Discrimination and Detection of Single Malaria Parasite Infected Erythrocytes on Glass. Faraday Discuss. 2016, 187, 341-352. https://doi.org/10.1039/c5fd00181a.

16. Wood, B. R.; Bambery, K. R.; Dixon, M. W. A.; Tilley, L.; Nasse, M. J.; Mattson, E.; Hirschmugl, C. J. Diagnosing Malaria Infected Cells at the Single Cell Level Using Focal Plane Array Fourier Transform Infrared Imaging Spectroscopy. Analyst 2014, 139 (19), 4769-4774. https://doi.org/10.1039/c4an00989d.

17. Lasch, P.; Naumann, D. Spatial Resolution in Infrared Microspectroscopic Imaging of Tissues. Biophys. Acta - Biomembr. 2006, 1758 (7), 814-829. 
https://doi.org/10.1016/j.bbamem.2006.06.008.

18. Bannister, L. H.; Hopkins, J. M.; Margos, G.; Dluzewski, A. R.; Mitchell, G. H. Three-Dimensional Ultrastructure of the Ring Stage of Plasmodium Falciparum: Evidence for Export Pathways. Microanal. 2004, 10 (5), 551-562. https://doi.org/10.1017/S1431927604040917.

19. Dluzewski, A. R.; Ling, I. T.; Hopkins, J. M.; Grainger, M.; Margos, G.; Mitchell, G. H.; Holder, A. A.; Bannister, L. H. Formation of the Food Vacuole in Plasmodium Falciparum. A Potential Role for the 19 KDa Fragment of Merozoite Surface Protein 1 (MSP119). PLoS One 2008, 3 (8). https://doi.org/10.1371/journal.pone.0003085.

20. Jackson, K. E.; Klonis, N.; Ferguson, D. J. P.; Adisa, A.; Dogovski, C.; Tilley, L. Food Vacuole-Associated Lipid Bodies and Heterogeneous Lipid Environments in the Malaria Parasite, Plasmodium Falciparum. Microbiol. 2004, 54 (1), 109-122. https://doi.org/10.1111/j.1365-2958.2004.04284.x.

21. Dazzi, A; Prater, C.B. AFM-IR: Technology and Applications in Nanoscale Infrared Spectroscopy and Chemical Imaging. Rev. 2017, 117 (7), 5146-5173. https://doi.org/10.1021/acs.chemrev.6b00448

22. Ruggeri, F. S.; Marcott, C.; Dinarelli, S.; Longo, G.; Girasole, M.; Dietler, G.; Knowles, T. P. J. Identification of Oxidative Stress in Red Blood Cells with Nanoscale Chemical Resolution by Infrared Nanospectroscopy. J. Mol. Sci. 2018, 19 (9), 1-15. https://doi.org/10.3390/ijms19092582.

23. Perez-Guaita, D.; Kochan, K.; Batty, M.; Doerig, C.; Garcia-Bustos, J.; Espinoza, S.; McNaughton, D.; Heraud, P.; Wood, B. R. Multispectral Atomic Force Microscopy-Infrared Nano-Imaging of Malaria Infected Red Blood Cells. Chem. 2018, 90 (5), 3140-3148.

https://doi.org/10.1021/acs.analchem.7b04318.

24. Kansiz, M.; Prater, C.; Dillon, E.; Lo, M.; Anderson, J.; Marcott, C.; Demissie, A.; Chen, Y.; Kunkel, G. Optical Photothermal Infrared Microspectroscopy with Simultaneous Raman - A New Non-Contact Failure Analysis Technique for Identification of $<10 \mathrm{Mm}$ Organic Contamination in the Hard Drive and Other Electronics Industries. Today 2020, 28 (3), 26-36. https://doi.org/10.1017/s1551929520000917.

25. Barth, A. Infrared Spectroscopy of Proteins. Biophys. Acta - Bioenerg. 2007, 1767 (9), 1073-1101. https://doi.org/10.1016/j.bbabio.2007.06.004.

26. Socrates, G. Infrared and Raman Characteristic Group Frequencies: Tables and Charts, 3rd ed.; Willey: New York, 2000.

27. Jaramillo, M.; Bellemare, M. J.; Martel, C.; Shio, M. T.; Contreras, A. P.; Godbout, M.; Roger, M.; Gaudreault, E.; Gosselin, J.; Bohle, D. S.; Olivier, M. Synthetic Plasmodium-like Hemozoin Activates the Immune Response: A Morphology - Function Study. PLoS One 2009, 4 (9). https://doi.org/10.1371/journal.pone.0006957.

28. Slater, A. F. G.; Swiggard, W. J.; Orton, B. R.; Flitter, W. D.; Goldberg, D. E.; Cerami, A.; Henderson, G. B. An Iron-Carboxylate Bond Links the Heme Units of Malaria Pigment. Natl. Acad. Sci. U. S. A. 1991, 88 (2), 325-329. https://doi.org/10.1073/pnas.88.2.325.

\section{Figures}




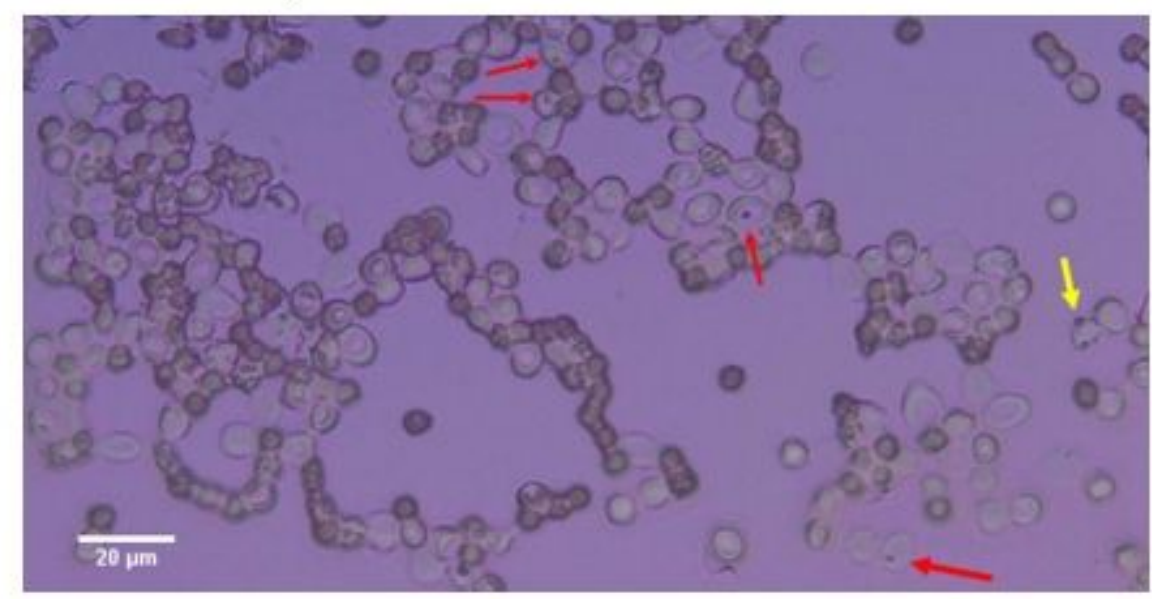

a

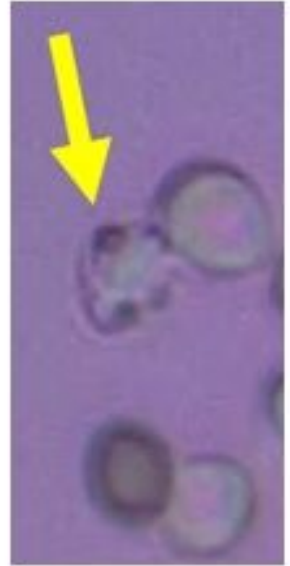

b

\section{Figure 1}

Example of plasmodium-infected RBCs, infected cells are marked by the red arrows; yellow arrow points for iRBC being a sample for experiments discussed in this work (a), enlarged area with cell of interest (b).

FTIR

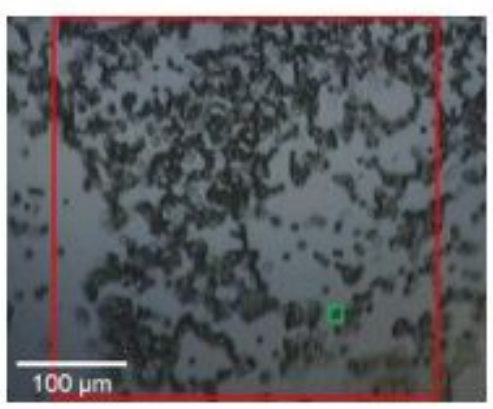

a

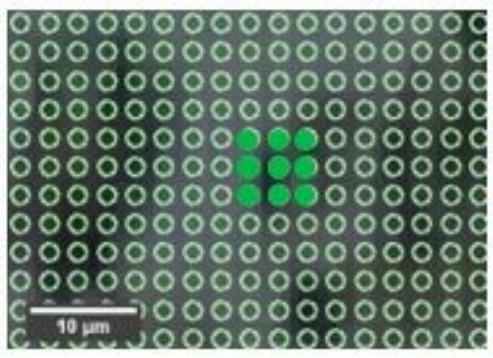

b

\section{O-PTIR}

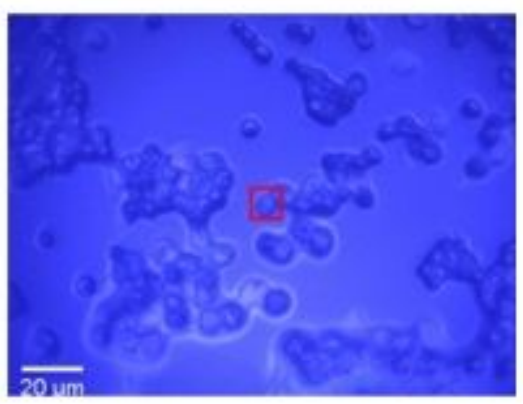

c

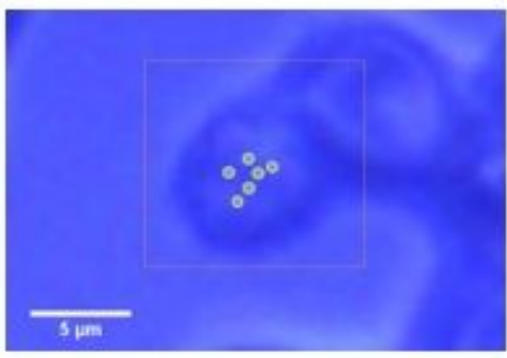

$\mathrm{d}$

\section{AFM-IR}

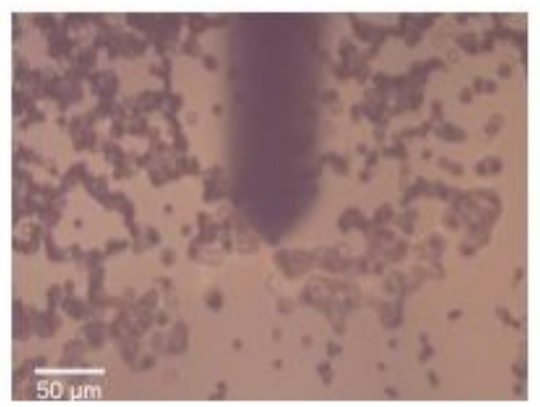

e

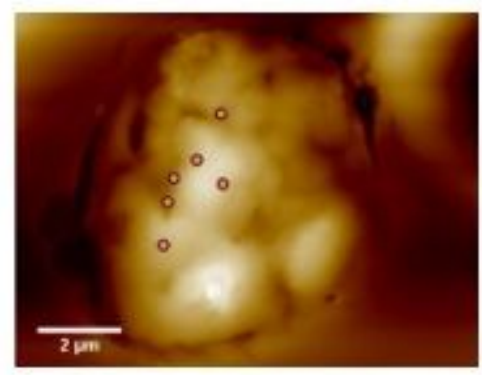

f

\section{Figure 2}

Screenshots from the various software provided by the manufacturers showing the area selected for the experiment for FTIR microspectroscopy (red square = one single FPA shot) (a), O-PTIR (c) and AFM-IR (e). iRBC of interest is marked by green square for FTIR (a) red square for O-PTIR (c); in case of AFM-IR the cantilever hides its exact localization (e). Zoomed region displaying part of $128 \times 128$ single elements of FPA representing pixels covering the area of selected iRBC (nine green circles) (b); red square on enlarged 
image presents the iRBC of interest, six blue circles show localization of O-PTIR spectra collection (d), AFM topographic image with six red circles denoting positions of spectra acquisition points for AFM-IR $(f)$.

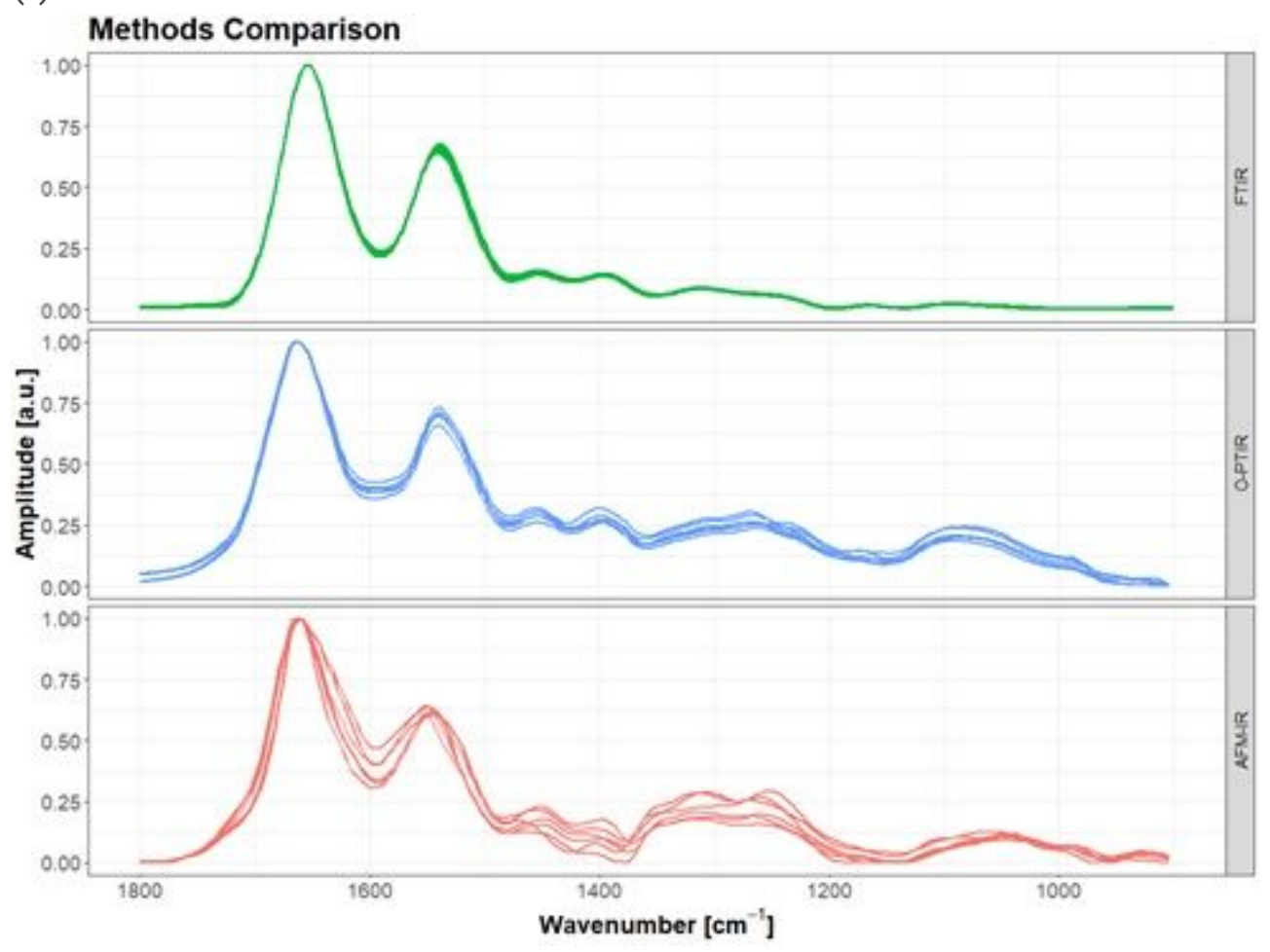

Figure 3

Colour-codded comparison of spectra taken for the same iRBC depending on the method in which they were collected (green line- FTIR, blue line-O-PTIR, red line - AFM-IR).

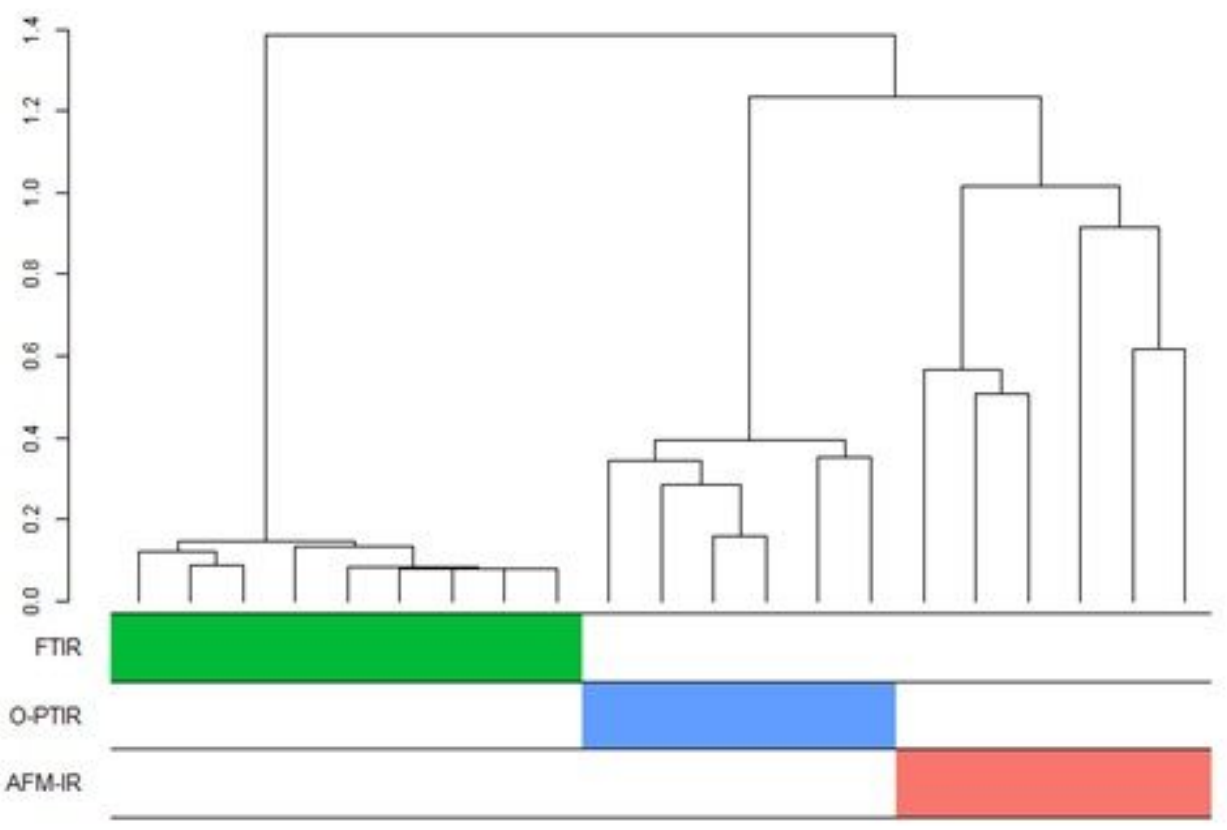

Figure 4 
Final results of HCA performed on spectra collected for the same iRBC by means of FTIR, O-PTIR and AFM-IR spectroscopies.
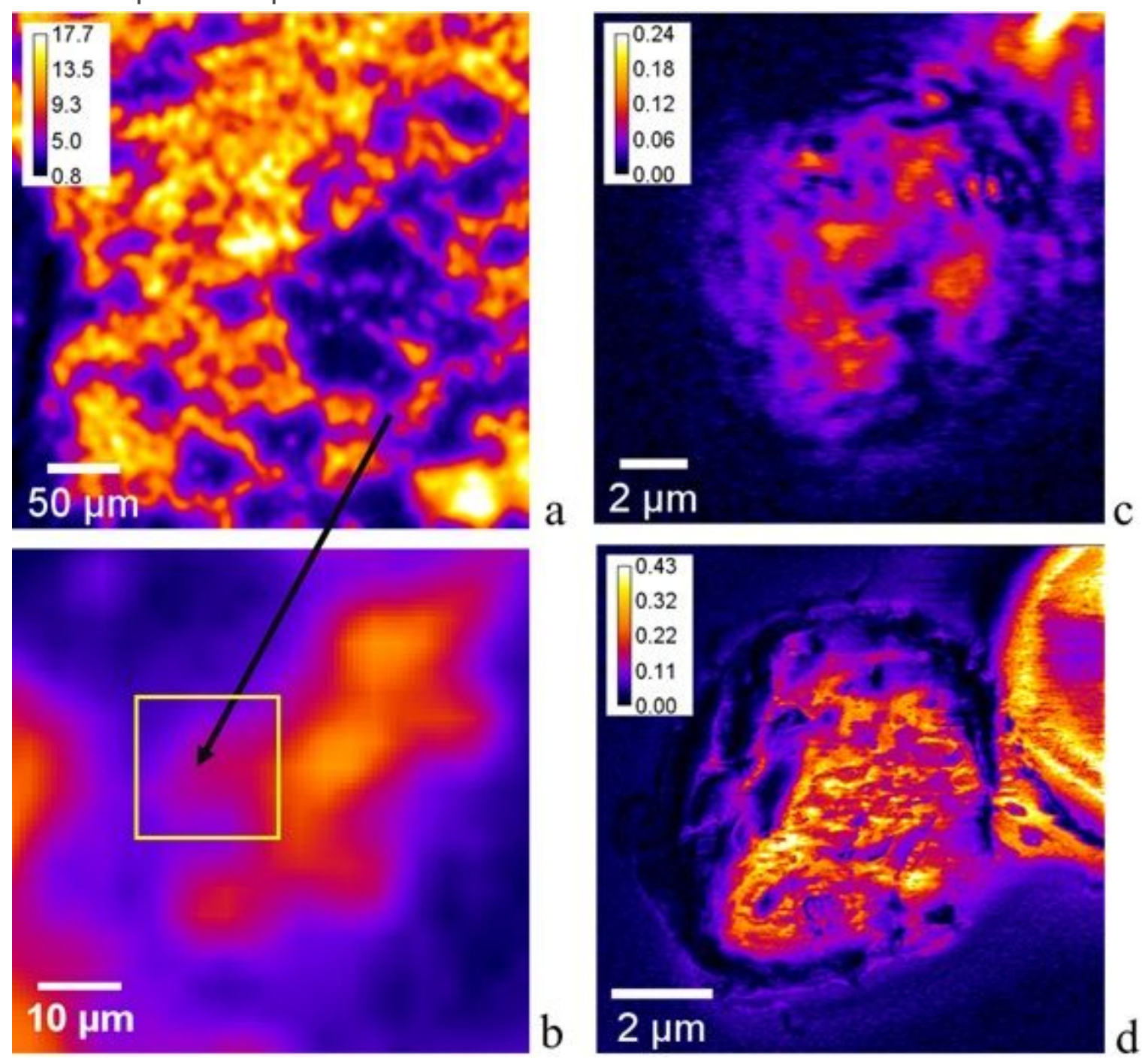

Figure 5

Distributions of Amide I, obtained during analysis of the same cell by means of FTIR microspectroscopy (a), O-PTIR (c) and AFM-IR (d). Yellow square in digitally zoomed area (b) from (a) around the cell of interest shows the localization of selected iRBC, blurred image cannot allow for noting any variation in Amide I distribution within single cells (FTIR microspectroscopy). 